\title{
DESIGN AND STUDY OF SWIMMING EXERCISE EQUIPMENT: STRENGTH TRAINING OF IMPROVING CORE MUSCLES
}

\author{
Dongdong Chang* \\ Tourism College of Zhejiang, Xiaoshan Higher Education Park, Hangzhou City, Zhejiang, 311231, China \\ E-mail: changdongdong@yahoo.com
}

\begin{abstract}
- as the technology develops, the mechanical-electrical integrated exercise equipment has been paid attentions to by more and more sports enthusiasts. In the swimming exercise, highquality training equipment can speed up the training progress, enhance and consolidate swimming skills, improve the strength training of the core muscles and strengthen the special quality of swimming. Currently, foreign core strength training equipment has many advantages because it adopts isokinetic equipment mode of exercise training which can completely simulate the underwater sports environment and realize real-time monitoring of training data while domestic one has deficiencies of imperfect mechanical structure design and unstable mechanical friction plus resistance output. On the basis of fully understanding the core muscle group and the core strength training theory, this paper carries out the design and research of swimming practice equipment. Through the absorption of foreign excellent experience, good core muscle training effect was achieved.
\end{abstract}

Keywords: swimming exercise equipment, core strength training, mechatronics, optical-electricity encoding.

\section{Introduction}

As a water sport, swimming has obvious geographical limitations. Professional swimming training not only includes water training but also contains land training using related training equipment, where the selection of equipment plays a key role. Many scholars have carried out related researches. Tong Junfang [1] designed the BK-1 swimming stool with electromagnetic resistance on the basis of a swimming stool developed by the US isokinetic strength training equipment company. Yan Li et al [2] used a set of hydraulic intelligent swimming test trainer with the functions of testing, diagnosing and training which accurately reflected the change of strength through measured data by sensors. Sun Jiali et al [3] developed a training method for athletes' lower limb strength, which replaced the previous squatting and single leg rubber pulling training methods.

In recent years, China's athletes have made remarkable achievements in the Olympic swimming projects, which is inseparable with the use of highquality swimming equipment. Studies showed that the core strength training could make the body react rapidly to changes during movement as well as help athletes make normative actions [4]. Moreover, it can reduce the resistance of fluid, save energy and decrease sport injury [5].

Good swimming exercise equipment can improve the strength training of core muscles and thus achieve better training results.

\section{Core strength training and swimming practice equipment}

\subsection{Theoretical study of core muscles and core strength training}

As shown in figure 1 , core muscles are muscle groups composed of rectus abdominis, musculus obliquus externus abdominis, lower back muscle and erector spinae, etc. [6]. They play a role of stabilizing the center of gravity and conducting strength.

Powerful core muscle groups can support body postures, exercise skills and skilled movements. The core strength training [7] aims to training the stability, strength and balance of body core muscles, as well as enhance the strength and endurance of core muscles [8]. Since the 1990's, the core strength training has been widely applied to the field of competitive sports as a new training theory. The core strength training can meet rapid strength requirements of decomposition, acceleration, deceleration, support and buffer, etc., of movements, which can help athletes better control their body movements during competition, improve muscle sense, save energy, relieve fatigue and reduce injury. 


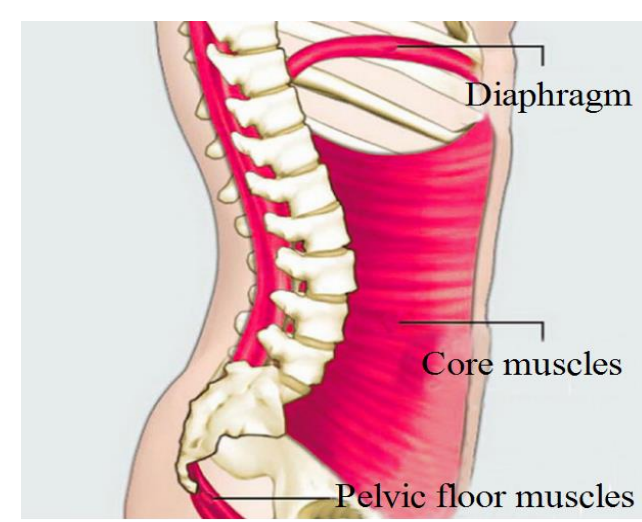

Figure 1:Sketch map of core muscle groups

\subsection{Application of core muscles and core strength training theories in swimming}

Through training the core muscles of body, the core strength training can gradually improve the training load of nerve muscles, enhance the muscle strength around joints, improve the control ability of nerve muscle system in core areas as well as improve the balance and stability of body during swimming. The application of strength training of core muscles in the filed of competitive sports greatly improves the training results [9]. The core strength training can effectively improve the cooperation level among muscles, reduce the load on small muscles and thus make the swimming movements more perfect.

\section{Design and study of equipment of core strength training of swimming}

\subsection{Core strength training equipment of swimming}

The core strength training equipment designed in this study is mainly used for freestyle swimming, as shown in figure 2.

The training equipment is composed of a connecting rod, a beam, a sliding plate, a wall pulley, a rotary encoder, a handrail, a swing frame, a podotheca, a mounting plate, an inertia wheel, a triangular belt, a rack, a tension sensor, an electric eddy current dynamometer, a measuring and control device, an information collector, a data converter and a computer.

An athlete lies on the mounting plate that is fixed and holds the handrail. He extends his hip joints and knees while his body is parallel to the floor. Then his feet are put on the foot frame and fixed by the podotheca. After that, the athlete begins the leg swing action of freestyle swimming.

The movement of feet can drive the swing of the swing frame and the connecting rod drives the crank rocker. Then the crank rocker drives the inertia wheel.
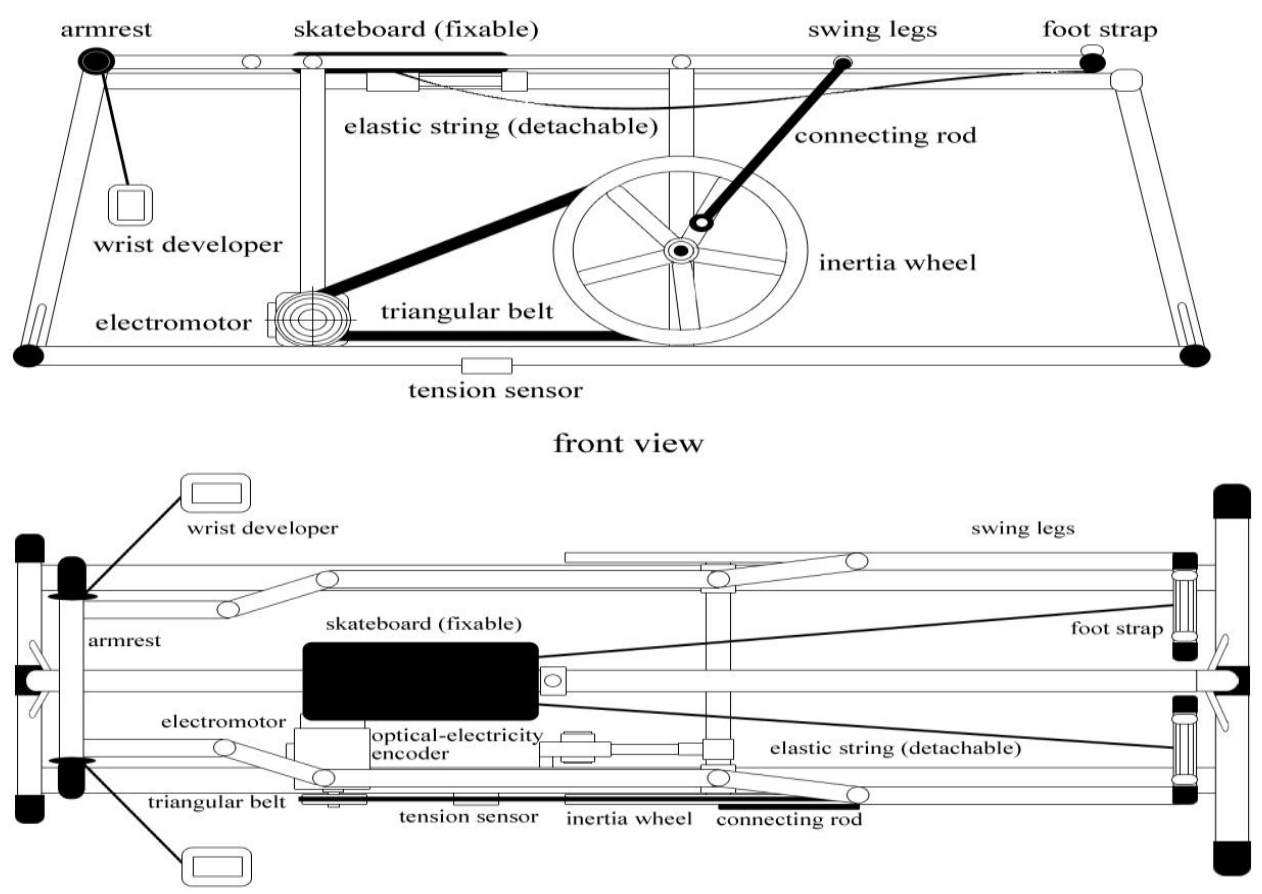

top view

Figure 2: Design scheme of the core strength training equipment of swimming 


\subsubsection{Design of the crank rocker}

The crank rocker [10] is composed of a rack, a connecting rode, a swing frame and an inertia wheel. The design of the crank rocker solves the problem of technical consistency and makes the reciprocating swing become circling motion. The size of the equipment is adjusted according to the average height of athletes and their exercise characteristics. The third of the swing frame is the length of the crank rocker. When the swing frame swings, the angles of the crank rocker are the same and the crank rocker does not have the character of quick-return motion.

\subsubsection{Design of the electric eddy current dynamometer}

The electric eddy current dynamometer [11] is a measurement test equipment that is widely applied at present, which is mainly composed of an eddy current retarder brake (rotor, vortex ring and field winding, etc.), dynamometry components, velocity measurement component and control system. The electric eddy current dynamometer absorbs power mainly through eddy-current loss. The network voltage is designed as around $200 \mathrm{~V}$ and $50 \mathrm{HZ}$; the output exciting voltage is current $30 \mathrm{~V}$ and the output exciting current is $0.5 \mathrm{~A}$.

When the direct current passes the magnet exciting coil, the magnetic flow is produced and it passes the vortex ring, air gap and rotor to form the magnetic line of force of closed loop circuit. The ankle of the athlete is connected with the rocking beam to drive the rotating disk. Due to the existence of eddy groove, the magnetic line of force is produced in the gap, thus the eddy potential is generated and the eddy current is formed. Meanwhile, the retarding torque is produced on the rotor and torque that is the same as the drive torque is produced on the swing body. The torque value can be detected by the sensor. The braking force is transmitted to the sensor during armature swing to measure the torque. The displacement signals are transformed into voltage signals through the transmitter and the value of torque can be calculated through analog-digital conversion.

\subsubsection{Design of the optical-electricity encoder}

The revolving speed is measured by the opticalelectricity encoder [12]. Because the photoelectric encoder and the motor are concentric, the raster plate revolves in the same speed as the motor. The measurement equipment that is composed of elements like luminous diode is used to measure pulse signals.
The calculation of the number of pulse output by the optical-electricity encoder every second can reflect the current revolving speed of the motor, thus the actual pedaling times of the athlete can be further revealed.

\section{Core Strength Trainer Test}

\subsection{Computer testing of swimming exercise equipment}

\subsubsection{Technical testing software}

Nowadays, a virtual instrument development software platform called VC8.0 is widely used in engineering testing and other fields, which is a standard data collection control software, with the advantages of visualization, data transfer, programming language structure, modular programming, fast running, fast test system, and strong reliability.

\subsubsection{Trainer test}

The test is carried out taking the chest developer as an example. Firstly, a photoelectric encoder is installed on the chest developer, which is connected to a notebook. Then, after entering the time period, the waveforms will appear and the relevant indicators will be displayed. The waveforms need to be preserved. Finally, data analysis is performed. According to the amplified waveforms, the athlete's waveform characteristics are analyzed and their shortcomings can be found.

\subsubsection{Waveform analysis of the chest developer}

In this study, the chest developer is integrated into a "mechatronics" [13] equipment mode. Through the analysis of the situation of the athletes and software feedback, targeted training is planned for the athletes. Theoretically speaking, the waveforms formed by swimmers during simulated pull are rectangular waves. That is, the fastest arm speed and the most standard action bring the fastest swimming speed and the most extensive coverage of the waveform. However, this ideal situation can hardly be realized in real situation. Nonetheless, through the waveform analysis and the situation analysis of the athletes, the most suitable training methods can be selected for athletes. 


\subsection{Actual measurement of the core strength exercising equipment}

\subsubsection{Objects and equipment of the actual measurement}

Objects of the actual measurement are 20 swimmers from a Shaanxi swimming team, in which 10 are males and 10 are females.

Five male swimmers and five female swimmers are formed into the experimental group randomly, while other 10 swimmers are the control group. Before the actual measurement, the health condition of swimmers is investigated to ensure that there is no injured athlete. Selected athletes should have similar training years. The measurement equipment are three equipment designed in this study (each one is equipped with a resistance sandbag) and necessary instruments like blood lactose tester were also equipped.

\subsubsection{Methods of actual measurement}

Leg swing of the freestyle swimming is used, and different training schemes are adopted respectively for the experiment group and the control group. The core strength training equipment is used for the strength training of the experiment group, while the control group adopts the original training method. The trainer operates as follows:
(1) Feet into the foot sets and fixed, hands grab the handrails, legs swing;

(2) Leg swing drives the inertia wheel rotation;

(3) With the right leg down and the left leg uplifting, the inertia wheel accelerates the crank, which forms a cycle.

The overall training time, group numbers, the amount of rest and the load degree of two groups are basically the same. Besides, the pulse monitor control index of training intensity should be the same, that is the training load should be the $60 \%$ of the maximum strength. The heart rate after the training is about 30 times every 10 seconds. Athletes in the experiment group have one hour of strength training using the core strength training equipment at $16 \sim 17$ o'clock in the afternoon every Monday to Friday. The amount of exercising includes two groups of 60 seconds of leg swing and two groups of 120 seconds of leg swing; the interval time between two group is 3 minutes. The training load of the control group is the same as that of the experiment group.

\subsubsection{Results of the actual measurement}

Experimental results show that, times of leg swing of athletes in the experiment group after using the core strength training equipment increase significantly. The increasing extent of 60 and 120 seconds of leg swing is $8.7 \%$ and $6.1 \%$ respectively. Therefore, the core strength training equipment has certain effects.

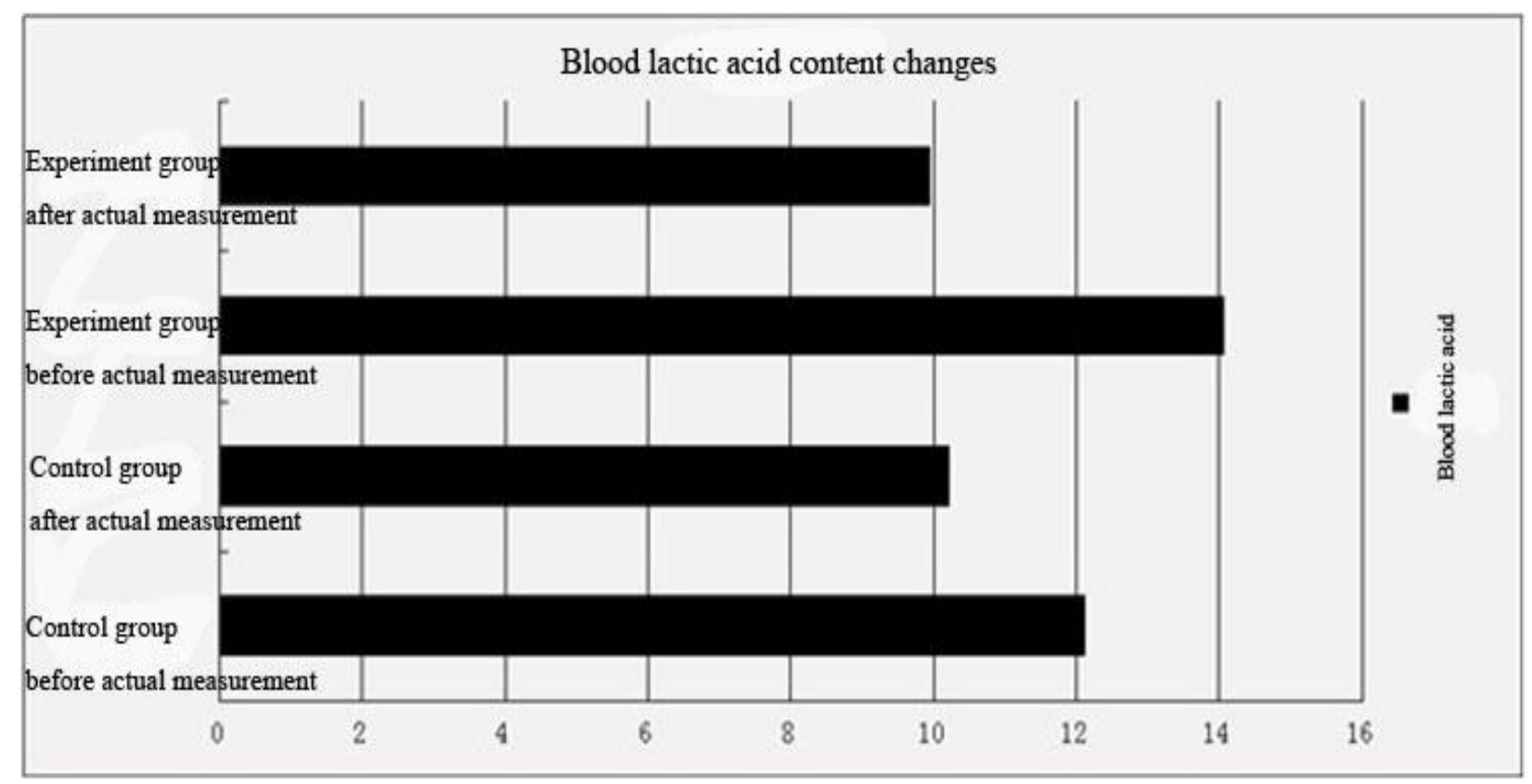

Figure 3: Changes of the blood lactic acid content before and after the actual measurement 


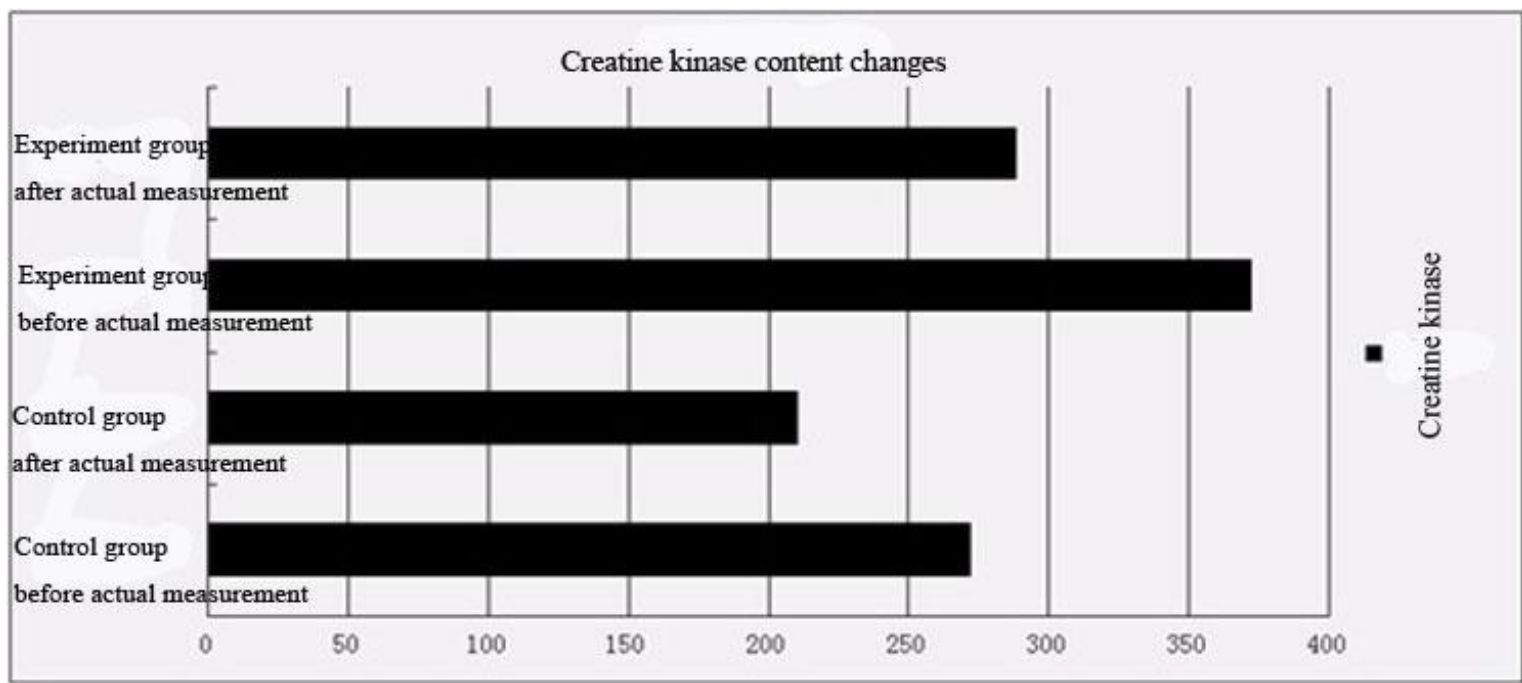

Figure 4: Changes of creatine kinase content before and after the actual measurement

As shown in figure 3 and figure 4, content changes of blood lactic acid and creatine kinase [14] show that changes of the experiment group is higher than that of the control group, indicating that the training intensity of the experiment group is higher.

Thus the training effect of the equipment is better than that of the traditional training.

\subsection{Athlete 's physical examination}

\subsubsection{Anaerobic endurance experiment of athletes}

Based on the former grouping, two teams were divided for bicycle aerobic [15] exercises after 10 weeks of strength training. The data were recorded and compared.

\subsubsection{Experimental results}

The results are shown in table 1.

Table 1 Comparison of endurance indexes during anaerobic exercise test

\begin{tabular}{|l|l|l|l|l|l|}
\hline \multicolumn{1}{|c|}{ Indexes } & \multicolumn{1}{|c|}{ group } & $\begin{array}{l}\text { Before } \\
\text { experiment }\end{array}$ & 3rd week & 5th week & 10 th week \\
\hline $\begin{array}{l}\text { Relative evaluation of } \\
\text { oxygenless work }\end{array}$ & Control group & $7.75 \mathrm{w} / \mathrm{kg}$ & $7.89 \mathrm{w} / \mathrm{kg}$ & $8 \mathrm{w} / \mathrm{kg}$ & $9.35 \mathrm{w} / \mathrm{kg}$ \\
\hline & $\begin{array}{l}\text { Experimental } \\
\text { grop }\end{array}$ & $7.78 \mathrm{w} / \mathrm{kg}$ & $8.03 \mathrm{w} / \mathrm{kg}$ & $8.35 \mathrm{w} / \mathrm{kg}$ & $9.88 \mathrm{w} / \mathrm{kg}$ \\
\hline Oxygen - free decay rate & $\begin{array}{l}\text { Control group } \\
\text { Experimental }\end{array}$ & $40.12 \%$ & $39.57 \%$ & $38.86 \%$ & $37.75 \%$ \\
\hline & $40.68 \%$ & $38.34 \%$ & $36.12 \%$ & $31.52 \%$ \\
\hline
\end{tabular}

As shown in table 1, after the swimming strength training, the athlete's relative anaerobic work was improved, while the oxygen-free power decay rate showed a significant decline. This proves that the training exercise improves the endurance of the athletes and their overall body quality.

\section{Discussion and Conclusions}

The core muscles and core strength are very important for the swimming training. With the support of core strength, the resistance of the body and arms and legs will be smaller, which is easier for athletes to complete relevant movements. Moreover, with the protection of core muscles, the stability of movement can be guaranteed, thus to reduce the probability of injury.

Currently, most instruments of core strength training are imported from foreign countries, which have great effects.

For example, the VASA isokinetic wall pulley from America, which has an adjustable air flow system that can simulate the resistance in water. Besides, it has a precise transmission shaft, a fan flywheel and a bearing, which can guarantee the smoothness of operation. Moreover, it is equipped with an electrical monitoring display. However, it is 
expensive and not perfect for the long-term mechanism of athletes.

Therefore, it is of great significance to develop better swimming equipment in China.

The designed core strength training equipment in this study learns the good experience and theories from foreign countries. The mechatronics mode system can collect exercising data during the exercises in real time, and data are output in the form of curves using special software. Besides, the advantages also include the simulation of resistance in water. Good-quality materials can reduce the impact force of traditional equipment on knees, thus to reduce the probability of injury. The swing frame, sliding plate and elastic string are adjustable, which increase the practical value of the training equipment. The whole designing process fully applies the ergonomic theory and the mechatronics technology, which makes the core strength training equipment be more humanized. The biggest advantage of the designed trainer lies in that it can reach the real situation to the largest extent and arranges proper training for the athletes in order to improve the endurance of the athletes. However, it is a little bit expensive for students and is not applicable in a wide range.

In conclusion, the application of core strength training equipment of swimming can improve the strength training of core muscles, enhance body strength, improve the control ability of body in water as well as save energy, thus to improve the swimming level of athletes.

\section{References}

[1] J. F. Tong, L. Kang. Design ideas and application of dynamic force swimming training device. Journal of Sports Science, 1988 (4): 76-79.

[2] L. Yang, et al. Study on Development of Dynamic Force Test Trainer for Hydraulic Intelligent Swimming. Journal of Wuhan Institute of Physical Education, 1998 (2): 82-86.

[3] J. L. Sun, et al. Study on development of lower limb strength training equipment for swimmers. 2001, 28 (5): 56-57.

[4] C. R. Lei, Q. H. Zou, F. F. Nie. Research on the Application of the Core Strength Training on the Men's 100-Meter Sprint Athletes Training Methods. Journal of Nanchang Normal University, 2014, 644-650:4864-4868.
[5] A. D. Faigenbaum, J. Schram. Can Resistance Training Reduce Injuries in Youth Sports. Strength \& Conditioning Journal, 2004, 26(3):16-21.

[6] B . Z. Niu. Application of core stability training in the field of rehabilitation medicine. The Journal of Medical Theory and Practice, 2015, (10):1300-1301.

[7] C. Luca, O. Lucio, Z. Emanuela, et al. Effects of different core exercises on respiratory parameters and abdominal strength. Journal of Physical Therapy Science, 2015, 27(10):32493253.

[8] F. Altug, T. C. Akman, N. Büker, et al. Effects of Two Different Muscle Strength Training Technique on Balance and Performance in Healthy Young People. Rawal Medical Journal, 2015, 40(2).

[9] N. Batalha, J. Marmeleira, N. Garrido, et al. Does a water-training macrocycle really create imbalances in swimmers' shoulder rotator muscles? European Journal of Sport Science, 2015, 6(1):1-6.

[10] X. Ma, Y. F. Gao. The Nature and Design of No Quick Return Peculiarity of Crank Rocker Mechanism. Applied Mechanics \& Materials, 2014, 602-605:29-32.

[11] M. C. Tsai, K. Y. Chiou, S. H. Wang, et al. Characteristics Measurement of Electric Motors by Contactless Eddy-Current Magnetic Coupler. Magnetics IEEE Transactions on, 2014, 50(11):1-4.

[12] R. Liu, R. Liu. Signal acquisition technology for photoelectric encoder based on FPGA. Optik International Journal for Light and Electron Optics, 2016, 127(20):9891-9895.

[13] Prof.PhD Eng. Gheorghe I. GHEORGHE, PhD Student Eng. Ilie Iulian. MECHATRONICS, INTEGRONICS \& ADAPTRONICS, EXCELLENCE IN THE FIELD OF INTELLIGENT SPECIALIZATION. Romanian Review Precision Mechanics, Optics \& Mechatronics, 2015, 47:17-21.

[14] Mougios V. Reference intervals for serum creatine kinase in athletes. British Journal of Sports Medicine, 2007, 41(41):674-678.

[15] Turley K R, Rivas J D, Townsend J R, et al. Effects of caffeine on anaerobic exercise in boys. Pediatric Exercise Science, 2012, 24(24):210219. 